\begin{tabular}{|l|l|}
\hline Title: & $\begin{array}{l}\text { Predictive Current Control of Saturated Cross-Coupled Permanent Magnet Synchronous } \\
\text { Machines }\end{array}$ \\
\hline Authors: & Jan Richter, Tobias Gemaßmer, Martin Doppelbauer \\
\hline Institute: & $\begin{array}{l}\text { Karlsruhe Institute of Technology (KIT) } \\
\text { Elektrotechnisches Institut (ETI) } \\
\text { Hybrid Electric Vehicles (HEV) }\end{array}$ \\
\hline Type: & Conference Proceedings \\
\hline Published at: & $\begin{array}{l}\text { 2014 International Symposium on Power Electronics, Electrical Drives, Automation and } \\
\text { Motion (SPEEDAM), Ischia, Italy, June 18-20, 2014 } \\
\text { Publisher: IEEE, Piscataway ( NJ) } \\
\text { Year: } 2014 \\
\text { ISBN: } 978-1-4799-4748-5 \\
\text { Pages: } 830-835\end{array}$ \\
\hline Hyperlinks: & DOI: $10.1109 /$ SPEEDAM.2014.6871930 \\
\hline
\end{tabular}

(c) 2014 IEEE. Personal use of this material is permitted. Permission from IEEE must be obtained for all other uses, in any current or future media, including reprinting/republishing this material for advertising or promotional purposes, creating new collective works, for resale or redistribution to servers or lists, or reuse of any copyrighted component of this work in other works. 


\title{
Predictive Current Control of Saturated Cross- Coupled Permanent Magnet Synchronous Machines
}

\author{
Jan Richter, Tobias Gemaßmer, Martin Doppelbauer \\ Karlsruhe Institute of Technology (KIT) \\ Elektrotechnisches Institut - Hybrid Electric Vehicles \\ Karlsruhe, Germany \\ Email: jan.richter@kit.edu
}

\begin{abstract}
Highly utilized permanent magnet synchronous machines show magnetic saturation and cross-coupling effects. These phenomena are described by nonlinear differential equations and make feedback current control of the machine challenging. State of the art current control methods usually ignore these effects and hence do not produce optimal results in transient operation or in operation at the inverter voltage limit. Therefore, this paper presents a method to design a current controller taking into account saturation and dynamic crosscoupling. Feed forward calculation of transient quantities in realtime enables complete decoupling and dead-beat behavior which is experimentally validated by test bench measurements. With this approach, strongly nonlinear, highly utilized synchronous machines can be successfully controlled achieving best control quality in all operational conditions.
\end{abstract}

Keywords-control design; cross-coupling; current control; machine vector control; nonlinear control systems; permanent magnet machines; predictive control; saturation magnetization

\section{INTRODUCTION}

Increasing power density of electric machines is a wide trend in modern machine development. It is driven by the need of small and light machines in the power regime of several tens of kilowatts e.g. for mobile applications. This is usually achieved by higher utilization of the machine's materials by increasing current and flux densities. As a consequence flux linkages as functions of machine currents become nonlinear. These physical phenomena have been extensively studied and are well understood. Thus nonlinear models of saturated, dynamically cross-coupled, magnetically anisotropic machines are available $[1,2]$ and employed in this contribution.

Numerous techniques to control permanent magnet synchronous machines have been developed. They differ in the controlled machine quantity (current control, direct torque control, direct speed control) and the control method (e.g. linear, hysteresis, fuzzy logic, sliding mode, predictive) $[3,4$, 5]. However, little has been done in incorporating machine nonlinearities like saturation and cross-coupling directly in respective control algorithms. An approximation using nonlinear stationary inductances in controlling synchronous reluctance motors was proposed by Morales-Caporal and Pacas [6]. Weigel and Mutschler linearize the motor equations within a control period and adjust control parameters according to the operation point [7]. Gemaßmer et al. achieved further improvement by prediction of the flux linkages of the subsequent control period for decoupling [8]. Nevertheless, in none of these approaches dynamic cross-coupling of the machine axes is considered. As a consequence the currents of the direct and quadrature axes remain dynamically coupled yielding unwanted current control deviations during transients. Hence, a modified rotor-oriented predictive current control method is presented that allows complete decoupling of the machine axes while taking into account iron saturation, dynamic cross-coupling and the voltage limit of the inverter. Thereby, full control of machine currents of both axes is achieved in all operational conditions although the machine behavior is strongly nonlinear.

The machine model is introduced in Section II. Subsequently, the current control algorithm (schematically shown in Fig. 1) is explained in detail. A description of the test bench and measurement setup for experimental evaluation of the controller's performance is given in Section III. According results are discussed in Section IV.

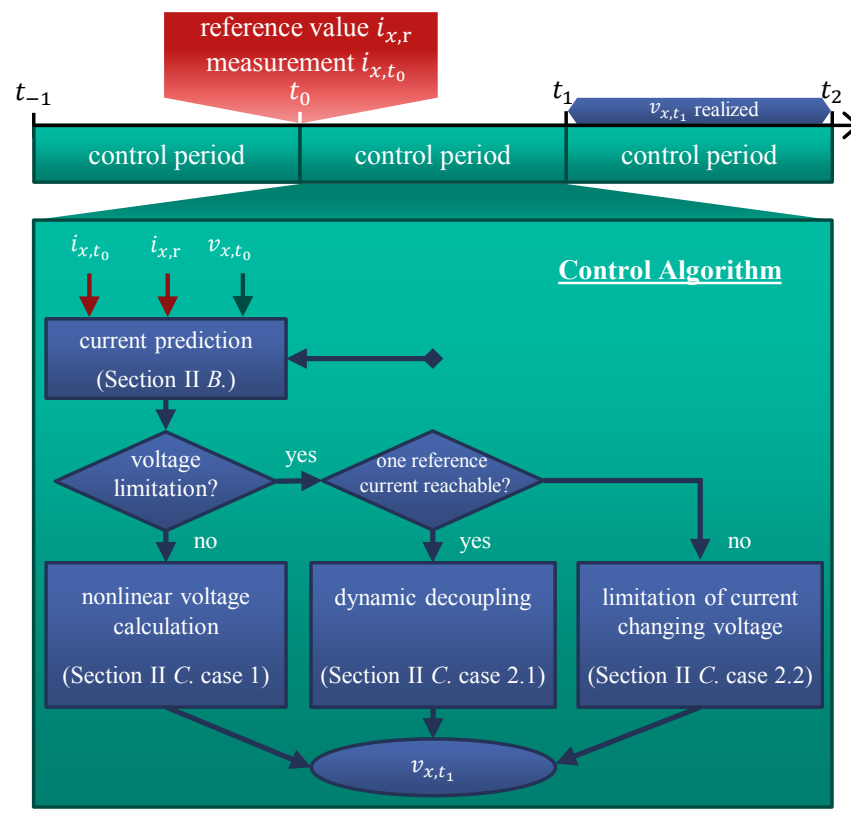

Fig. 1. Current control algorithm. The variable $x$ represents the direct and quadrature axis $(x \in\{\mathrm{d}, \mathrm{q}\})$. 


\section{THEORY}

The ideal approach to control a device described by a system of nonlinear differential equations is to compute a complete solution at each time step, taking all relevant physical effects into account. If such a calculation could be done in real-time, an ideal controller could be designed. Even though this is not possible with today's microprocessors, nevertheless one should stick with the original equations as accurately as possible. By this approach it can be shown that not the currents themselves but the flux linkages can be directly predicted. These again can be uniquely mapped to the currents, which will be proven to hold true for any given synchronous machine. Thereby the current control deviation after every control period can be predicted and an according strategy to minimize the control deviation can be designed.

\section{A. Machine Model}

A machine with three symmetric, star-connected phases with the neutral point not connected to the inverter is assumed. Dielectric currents, spatial air-gap harmonics and temperature effects are neglected. By employing Faraday's law of induction, Ohm's law and Kirchhoff's laws to the machine coils and subsequent transformation to the rotor-fixed dq-frame [2] the stator voltage can be expressed as

$$
\begin{aligned}
& v_{\mathrm{d}}=R i_{\mathrm{d}}+\frac{\mathrm{d} \Psi_{\mathrm{d}}}{\mathrm{d} t}-\omega \Psi_{\mathrm{q}} \\
& v_{\mathrm{q}}=R i_{\mathrm{q}}+\frac{\mathrm{d} \Psi_{\mathrm{q}}}{\mathrm{d} t}+\omega \Psi_{\mathrm{d}} .
\end{aligned}
$$

There $R$ denotes the stator resistance, $t$ the time, $\omega$ the electric angular frequency and $v_{x}, i_{x}$ and $\Psi_{x}$ the voltages, currents and flux linkages of the direct and quadrature axis $(x \in\{\mathrm{d}, \mathrm{q}\})$ with the direct axis being aligned to the permanent magnets. Equations (1) and (2) are coupled through the rotary induced voltage $\omega \Psi_{x}$ and through self and mutual induction $\frac{\mathrm{d} \Psi_{x}}{\mathrm{~d} t}$ because the flux linkages depend on the currents of both axes to incorporate saturation and cross-coupling. The relation between the currents and flux linkages is defined as

$$
f: \mathbb{R}^{2} \rightarrow \mathbb{R}^{2},\left(i_{\mathrm{d}}, i_{\mathrm{q}}\right) \mapsto\left(\Psi_{\mathrm{d}}, \Psi_{\mathrm{q}}\right)
$$

consisting of two components $f:=\left(f_{\Psi_{d}}, f_{\Psi_{q}}\right)$ both being nonlinear two-dimensional functions. One-dimensional functions are invertible if the first derivative is greater than zero in the considered interval. For multi-dimensional functions this can be generalized to the Jacobian determinant being greater than zero in the area to be inverted. Applied to $f$ this yields

$$
\underbrace{\frac{\partial f_{\Psi_{\mathrm{d}}}}{\partial i_{\mathrm{d}}} \cdot \frac{\partial f_{\Psi_{\mathrm{q}}}}{\partial i_{\mathrm{q}}}}_{\text {self-induction }}-\underbrace{\frac{\partial f_{\Psi_{\mathrm{d}}}}{\partial i_{\mathrm{q}}} \cdot \frac{\partial f_{\Psi_{\mathrm{q}}}}{\partial i_{\mathrm{d}}}}_{\text {cross-coupling }}>0,
$$

whereas the minuend represents self-induction and the subtrahend cross-coupling. Self-induction partial derivatives are always greater than zero which is directly implied by Ampère's circuital law. Cross-coupling partial derivatives are greater or smaller than zero depending on the point of operation. As the effect of cross-coupling can never exceed the effect of self-induction in real systems with magnetic leakage (mutual induction is smaller than self-induction due to Gauss's

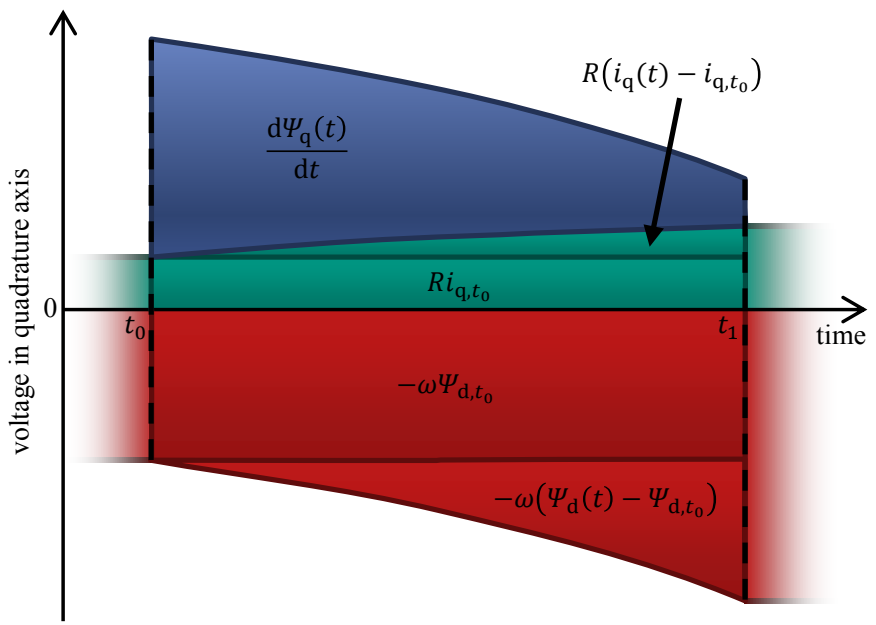

Fig. 2 Stationary and dynamic components of the quadrature axis voltage during one control period (not true to scale)

law for magnetism), the product of the cross-coupling derivatives is always smaller than the product of self-induction derivatives. Hence the Jacobian determinant is always greater than zero and $f$ can be inverted.

$$
f^{-1}: \mathbb{R}^{2} \rightarrow \mathbb{R}^{2},\left(\Psi_{\mathrm{d}}, \Psi_{\mathrm{q}}\right) \mapsto\left(i_{\mathrm{d}}, i_{\mathrm{q}}\right)
$$

\section{B. Current Prediction}

The calculation of the control algorithm requires a certain amount of time. Thus currents can only be measured at $t_{0}$ before starting the calculation (see Fig. 1). However, optimal control performance can still be achieved as long as the voltages calculated by the control algorithm consider variations of the motor quantities in the interval $\left[t_{0}, t_{1}\right]$. Thus the currents at $t_{1}$ need to be predicted from information available at $t_{0}$. The necessary voltages in the interval $\left[t_{0}, t_{1}\right]$ are calculated by the control algorithm in the foregoing interval $\left[t_{-1}, t_{0}\right]$ and denoted $v_{x, t_{0}}$ in the following. These voltages and the measured currents are then inserted into the system of differential equations to predict the flux linkages (which can be mapped to the currents). For this, (1) and (2) are transformed into a time-discrete form as exemplified for the quadrature axis in Fig. 2. There the voltage-time areas of the different quadrature voltage components are displayed in the interval $\left[t_{0}, t_{1}\right]$. It can be seen that both currents and flux linkages increase nonlinearly. In order to predict the flux linkages at $t_{1}$, either a time-step simulation has to be employed or the dynamic ohmic voltage drop $R\left(i_{\mathrm{q}}(t)-i_{\mathrm{q}, t_{0}}\right)$ must be neglected and the trajectories of the flux linkages assumed to be linear. As time-step simulations are hardly real-time capable, this approach is omitted. Instead, the validity of the proposed approximations is shown in Section IV. Employing these approximations yields

$$
\begin{aligned}
& v_{\mathrm{d}, t_{0}}=R i_{\mathrm{d}, \mathrm{t}_{0}}+\frac{\Psi_{\mathrm{d}, t_{1}}-\Psi_{\mathrm{d}, t_{0}}}{T_{\mathrm{A}}}-\frac{1}{2} \omega\left(\Psi_{\mathrm{q}, t_{0}}+\Psi_{\mathrm{q}, t_{1}}\right) \\
& v_{\mathrm{q}, t_{0}}=R i_{\mathrm{q}, \mathrm{t}_{0}}+\frac{\Psi_{\mathrm{q}, t_{1}}-\Psi_{\mathrm{q}, t_{0}}}{T_{\mathrm{A}}}+\frac{1}{2} \omega\left(\Psi_{\mathrm{d}, t_{0}}+\Psi_{\mathrm{d}, t_{1}}\right)
\end{aligned}
$$

with $T_{\mathrm{A}}=t_{1}-t_{0}$ denoting the sampling time. The flux linkages $\Psi_{\mathrm{d}, t_{0}}$ and $\Psi_{\mathrm{q}, t_{0}}$ are calculated by (3) using the 
measured currents $i_{\mathrm{d}, t_{0}}$ and $i_{\mathrm{q}, t_{0}}$. The electrical frequency $\omega$ is assumed to be constant during the control period which is a valid assumption for most drive systems because mechanical time constants exceed $T_{\mathrm{A}}$ by several orders of magnitude. Solving (6) and (7) for the predicted flux linkages $\Psi_{\mathrm{d}, t_{1}}$ and $\Psi_{\mathrm{q}, t_{1}}$ yields

$$
\begin{aligned}
\Psi_{\mathrm{d}, t_{1}} & =\Psi_{\mathrm{d}, t_{0}}+T_{\mathrm{A}} \cdot \frac{v_{\mathrm{d}, t_{0}}-R \cdot i_{\mathrm{d}, t_{0}}+\omega \Psi_{\mathrm{q}, t_{0}}}{1+\frac{1}{4} \omega^{2} T_{\mathrm{A}}^{2}} \\
& +T_{\mathrm{A}}^{2} \cdot \frac{\frac{1}{2} \omega v_{\mathrm{q}, t_{0}}-\frac{1}{2} \omega R i_{\mathrm{q}, t_{0}}-\frac{1}{4} \omega^{2} \Psi_{\mathrm{d}, t_{0}}}{1+\frac{1}{4} \omega^{2} T_{\mathrm{A}}^{2}} \\
\Psi_{\mathrm{q}, t_{1}} & =\Psi_{\mathrm{q}, t_{0}}+T_{\mathrm{A}} \cdot \frac{v_{\mathrm{q}, t_{0}}-R \cdot i_{\mathrm{q}, t_{0}}-\omega \Psi_{\mathrm{d}, t_{0}}}{1+\frac{1}{4} \omega^{2} T_{\mathrm{A}}^{2}} \\
+ & T_{\mathrm{A}}^{2} \cdot \frac{-\frac{1}{2} \omega v_{\mathrm{d}, t_{0}}+\frac{1}{2} \omega R i_{\mathrm{d}, t_{0}}-\frac{1}{4} \omega^{2} \Psi_{\mathrm{q}, t_{0}}}{1+\frac{1}{4} \omega^{2} T_{\mathrm{A}}^{2}}
\end{aligned}
$$

The predicted currents are finally obtained by using the inverse function given in (5).

$$
\left(i_{\mathrm{d}, t_{1}}, i_{\mathrm{q}, t_{1}}\right)=f^{-1}\left(\Psi_{\mathrm{d}, t_{1}}, \Psi_{\mathrm{q}, t_{1}}\right)
$$

\section{Control Algorithm}

The control algorithm optimizes the inverter output phase voltage in order to quickly and precisely follow the given current reference values. In order to illustrate the principle of the control algorithm, Fig. 3 is analyzed. There, the function $\Psi_{\mathrm{q}}=f_{\Psi_{\mathrm{q}}}\left(i_{\mathrm{d}}, i_{\mathrm{q}}\right)$ is shown which relates both currents to the quadrature flux linkage. Starting with the measured currents, the currents at $t_{1}$ are predicted by (8) to (10) and marked by the white cross. The black shape shows the current combinations that can be reached at $t_{2}$ when considering the limited output voltage of the inverter. Due to real-time constraints the black shape cannot be computed in total by the control algorithm. It should be noted that this calculation is not even necessary because optimal inverter output voltage can be directly derived as will be shown in the following. Depending on the values of the measured and the reference currents three cases can be distinguished:

- Both reference values lie within the black shape and can be reached at $t_{2}$ (case 1 ).

- One reference current can be reached at $t_{2}$ (case 2.1).

- None of the reference currents can be reached at $t_{2}$ (case 2.2).

At first, the relevant case has to be identified. Therefore the flux linkages corresponding to the reference currents

$$
\left(\Psi_{\mathrm{d}, \mathrm{r}}, \Psi_{\mathrm{q}, \mathrm{r}}\right)=f\left(i_{\mathrm{d}, \mathrm{r}}, i_{\mathrm{q}, \mathrm{r}}\right)
$$

are calculated. By inserting the latter as well as all reference values into (6) and (7), the voltages to be applied at $t_{1}$ to reach the reference currents at $t_{2}$ can then be calculated.

$$
\begin{aligned}
& v_{\mathrm{d}, \mathrm{r}, t_{1}}=R i_{\mathrm{d}, t_{1}}+\frac{\Psi_{\mathrm{d}, \mathrm{r}}-\Psi_{\mathrm{d}, t_{1}}}{T_{\mathrm{A}}}-\frac{1}{2} \omega\left(\Psi_{\mathrm{q}, t_{1}}+\Psi_{\mathrm{q}, \mathrm{r}}\right) \\
& v_{\mathrm{q}, \mathrm{r}, t_{1}}=R i_{\mathrm{q}, t_{1}}+\frac{\Psi_{\mathrm{q}, \mathrm{r}}-\Psi_{\mathrm{q}, t_{1}}}{T_{\mathrm{A}}}+\frac{1}{2} \omega\left(\Psi_{\mathrm{d}, t_{1}}+\Psi_{\mathrm{d}, \mathrm{r}}\right)
\end{aligned}
$$

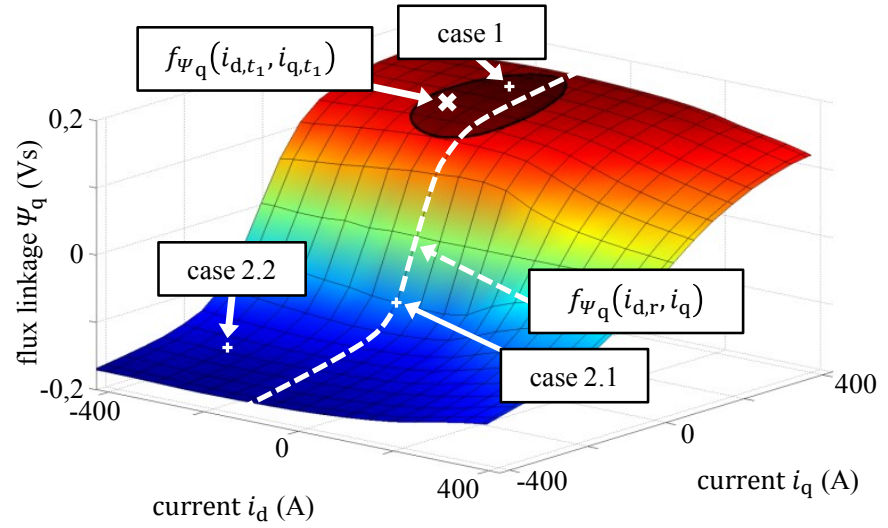

Fig. 3 Function $\Psi_{\mathrm{q}}=f_{\Psi_{\mathrm{q}}}\left(i_{\mathrm{d}}, i_{\mathrm{q}}\right)$ for the machine under test determined through measurements as described in Section III $B$. A point of operation with $i_{\mathrm{d}, t_{1}}$ being $-200 \mathrm{~A}$ and $i_{\mathrm{q}, t_{1}}$ being $200 \mathrm{~A}$ is exemplarily marked by a white cross. The black shape shows the reachable current combinations and quadrature flux linkage within one control period at $2000 \mathrm{~min}^{-1}$ using a DC link voltage of $300 \mathrm{~V}$.

Case 1 and 2 can be distinguished by evaluating (12) and (13) and checking if the maximum inverter output phase voltage $v_{\text {max }}$ is exceeded

$$
\sqrt{v_{\mathrm{d}, t_{1}}^{2}+v_{\mathrm{q}, t_{1}}^{2}} \leq v_{\max }(\text { case } 1)
$$

In case 1 , control deviations of both currents can be removed within one control period by applying the voltages from (12) and (13) yielding dead-beat behavior.

In case 2 , the maximum inverter output phase voltage $v_{\max }$ is not high enough to remove the control deviations of both currents within one control period. The maximum possible output voltage should be applied, but the distribution of the voltage to the direct and quadrature axis has yet to be determined. Again, the control strategy depends on the relevant case. For distinction of case 2.1 and 2.2 it needs to be checked if at least one reference current can be reached within the control period. Therefore the current changing voltage component is solely applied to one axis. This is calculated by evaluation of (12) and (13) and inserting the reference value of one component and the predicted value of the other. By checking (14) one can then distinguish if at least one reference value can be reached within one control period (case 2.1) or not (case 2.2).

In case 2.1 the control strategy is to precisely reach one reference value while the other one is approached as close as possible, thereby enabling dynamic decoupling. Since it is known that one reference value is reached, its value at the end of the subsequent control period at $t_{2}$ is known: It is the corresponding reference value. The absolute value of the voltage is the maximum inverter output phase voltage $v_{\text {max }}$. This knowledge is sufficient to correctly calculate both voltage components $v_{\mathrm{d}, \mathrm{lim} 2.1}$ and $v_{\mathrm{q}, \mathrm{lim} 2.1}$. The voltages are connected by

$$
v_{\max } \stackrel{!}{=} \sqrt{v_{\mathrm{d}, \lim 2.1}^{2}+v_{\mathrm{q}, \lim 2.1}^{2}}
$$




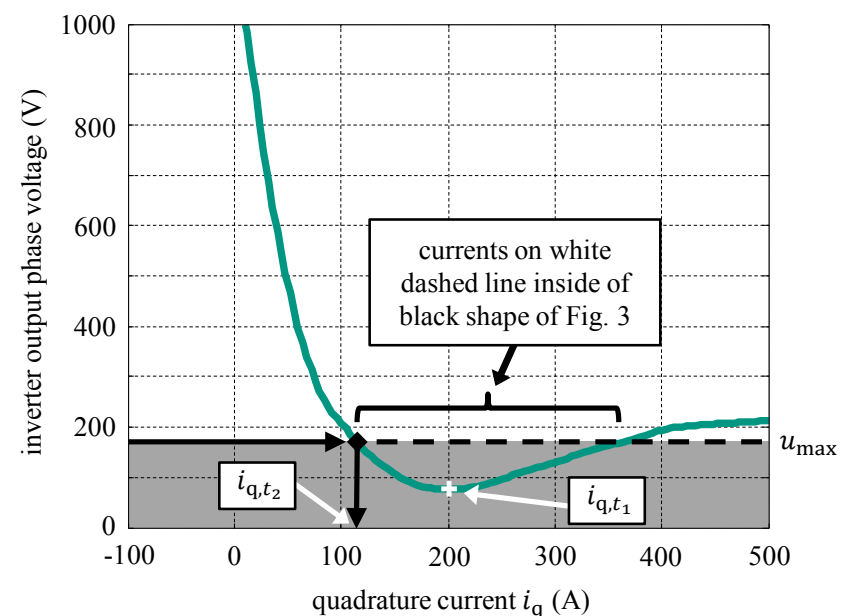

Fig. 4. Determination of the current at the end of the subsequent control period in case 2.1. The determined intersection of this figure corresponds to the lower intersection point between the thick black and white dotted line in Fig. 3. The maximum inverter output phase voltage $v_{\max }$ is $173.2 \mathrm{~V}$ at a DC link voltage of $300 \mathrm{~V}$ if space vector modulation is applied.

Moreover since one current at $t_{2}$ is known, the functions $f_{\Psi_{\mathrm{d}}}$ and $f_{\Psi_{\mathrm{q}}}$ both become one-dimensional. This is exemplarily shown in Fig. 3 for $f_{\Psi_{\mathrm{q}}}\left(i_{\mathrm{d}, \mathrm{r}}, i_{\mathrm{q}}\right)$ by the thick dashed white line. Therefore by employing (12) and (13) the voltages $v_{\mathrm{d}, \lim 2.1}$ and $v_{\mathrm{q}, \mathrm{lim} 2.1}$ can be written as

$$
\begin{aligned}
v_{\mathrm{d}, \lim 2.1}=R i_{\mathrm{d}, t_{1}} & +\frac{f_{\Psi_{\mathrm{d}}}\left(i_{\mathrm{d}, \mathrm{r}}, i_{\mathrm{q}}\right)-\Psi_{\mathrm{d}, t_{1}}}{T_{\mathrm{A}}} \\
& -\frac{1}{2} \omega\left(\Psi_{\mathrm{q}, t_{1}}+f_{\Psi_{\mathrm{q}}}\left(i_{\mathrm{d}, \mathrm{r}}, i_{\mathrm{q}}\right)\right) \\
v_{\mathrm{q}, \lim 2.1}=R i_{\mathrm{q}, t_{1}} & +\frac{f_{\Psi_{\mathrm{q}}}\left(i_{\mathrm{d}, \mathrm{r}}, i_{\mathrm{q}}\right)-\Psi_{\mathrm{q}, t_{1}}}{T_{\mathrm{A}}} \\
& +\frac{1}{2} \omega\left(\Psi_{\mathrm{d}, \mathrm{t}_{1}}+f_{\Psi_{\mathrm{d}}}\left(i_{\mathrm{d}, \mathrm{r}}, i_{\mathrm{q}}\right)\right)
\end{aligned}
$$

while all variables but $i_{\mathrm{q}}$ are known. By substitution of (16) and (17) into (15) the needed absolute value of the inverter output phase voltage in dependence of $i_{\mathrm{q}}$ is obtained. This is shown in Fig. 4 by the green curve. Intersection with the maximum inverter output phase voltage yields the reachable quadrature current $i_{\mathrm{q}, t_{2}}$ at $t_{2}$. The voltages can then again be calculated with (12) and (13), obtaining

$$
\begin{aligned}
& v_{\mathrm{d}, 2.1, t_{1}}=R i_{\mathrm{d}, t_{1}}+\frac{\Psi_{\mathrm{d}, t_{2}}-\Psi_{\mathrm{d}, t_{1}}}{T_{\mathrm{A}}}-\frac{1}{2} \omega\left(\Psi_{\mathrm{q}, t_{1}}+\Psi_{\mathrm{q}, t_{2}}\right) \\
& v_{\mathrm{q}, 2.1, t_{1}}=R i_{\mathrm{q}, t_{1}}+\frac{\Psi_{\mathrm{q}, t_{2}}-\Psi_{\mathrm{q}, t_{1}}}{T_{\mathrm{A}}}+\frac{1}{2} \omega\left(\Psi_{\mathrm{d}, t_{1}}+\Psi_{\mathrm{d}, t_{2}}\right)
\end{aligned}
$$

Using this method, one current reaches its reference value while the other approaches its reference value as close as possible. This results in a dynamic decoupling of the machine axes in both transient and stationary operation.

When none of the reference currents can be reached within the control period (case 2.2) the current-changing voltage component is limited to the maximal possible value (see Fig. 5). This ensures sufficient voltage for stationary operation

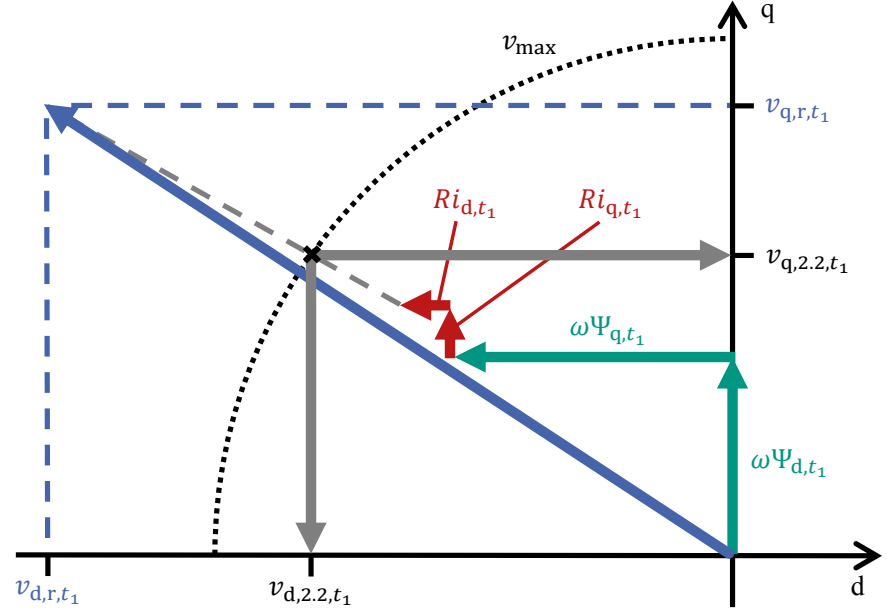

Fig. 5 Procedure to calculate the output voltage by limitation of the current changing voltage component if none of the reference currents can be reached within one control period (case 2.2).

while the more voltage is allocated to one axis the more is needed to reach the respective reference value. The procedure to calculate the voltages $v_{\mathrm{d}, 2.2, t_{1}}$ and $v_{\mathrm{q}, 2.2, t_{1}}$ by determination of the intersection of the current changing voltage and the circle of maximum inverter output phase voltage is illustrated in Fig. 5. The problem can be reduced to solving a quadratic equation $[8,9]$.

The proposed control strategy works very well as long as the motor parameters are known precisely. However, model parameters like the ohmic resistance or the flux linkages change during operation. Thus an integral path is added but only activated when the measured currents are close to the reference values to achieve stationary accuracy.

\section{EXPERIMENTAL}

\section{A. Test Bench and Measurement Equipment}

Test bench measurements are conducted with a permanentmagnet reluctance torque synchronous machine of type Brusa HSM1-6.1712-CO1. The machine's characteristics are given in Table 1. An asynchronous machine by Wittur is used as load. Inverters for grid connection and control of both machines switching at $8 \mathrm{kHz}$ are based on Semikron SkiiP 513GD122$3 D U L$ modules. In order to operate the machine with nominal voltage a DC link voltage of $300 \mathrm{~V}$ and space vector

TABLE I. MACHINE PROPERTIES

\begin{tabular}{|l|c|}
\hline \multicolumn{1}{|c|}{ Parameter } & Nominal Values \\
\hline Voltage nom. & $212 \mathrm{~V}$ \\
\hline Current nom. / max. & $169 \mathrm{~A} / 300 \mathrm{~A}$ \\
\hline Shaft power nom. / max. & $57 \mathrm{~kW} / 97 \mathrm{~kW}$ \\
\hline Speed nom. / max. & $4200 \mathrm{~min}^{-1} / 11000 \mathrm{~min}^{-1}$ \\
\hline Torque nom. / max. & $130 \mathrm{Nm} / 220 \mathrm{Nm}$ \\
\hline Ohmic stator resistance typ. & $10.5 \mathrm{~m} \Omega$ \\
\hline Number of pole pairs & 3 \\
\hline
\end{tabular}


modulation is used. Currents are measured by the built-in Semikron SkiiP current transducers. Torque is observed with a HBM T1OF torque meter. Speed and rotor angle are calculated using the machine's built-in incremental encoder signals. For central data capturing a digital signal processor system is used that records line voltages, phase currents, torque, speed and rotor angle data with a sampling rate of $8 \mathrm{kHz}$.

\section{B. Identification of Parameters}

Machine parameters are determined using stationary measurements. If the machine is current-controlled to constant direct and quadrature currents the dynamic terms $\frac{\mathrm{d} \Psi_{\mathrm{d}}}{\mathrm{d} t}$ and $\frac{\mathrm{d} \Psi_{\mathrm{q}}}{\mathrm{d} t}$ in (1) and (2) are zero and the equations can be solved for $\Psi_{\mathrm{d}}$ and $\Psi_{\mathrm{q}}$. By variation of the currents of both axes the characteristic flux linkage diagrams like the one for $\Psi_{\mathrm{q}}$ in Fig. 3 can be identified.

\section{Calculation of the Control Algorithm}

The control algorithm described in Section II is implemented on the digital signal processor (DSP) TMS320C6748 produced by Texas Instruments. The lookup tables of the parameter fields of the flux linkages are stored in an external SD-RAM and require 2 megabytes in total. To realize a switching frequency of $8 \mathrm{kHz}$ all control algorithm calculations are executed within less than $125 \mu$ s. Inverter gate signals are created by a modulator using a field programmable gate array (FPGA) of the Cyclone series by Altera.

\section{RESULTS AND DISCUSSION}

\section{A. Validity of Approximations}

In the derivations of Section II $B$ it was claimed that the dynamic ohmic voltage drop can be neglected and that the flux linkage trajectory can be assumed to be linear. The first statement is valid because ohmic voltage losses are small for the machine under test. By neglecting the ohmic resistance the resulting prediction error of the currents during one control period can be estimated. Therefore, the smallest differential inductance of the machine is assumed to be valid during the whole control period (worst-case scenario). In the allowed area of operation the machine exhibits its lowest differential inductance in its direct axis.

$$
L_{\mathrm{d}, \mathrm{diff}, \min }=\min \left(\frac{\partial f_{\Psi_{\mathrm{d}}}}{\partial i_{\mathrm{d}}}\right)=16.5 \mu \mathrm{H}
$$

This yields a time constant of

$$
\tau=\frac{L_{\mathrm{d}, \mathrm{diff}, \min }}{R}=1.6 \mathrm{~ms} .
$$

The difference between considering and neglecting the ohmic resistance can be calculated by assuming the current to increase from $0 \mathrm{~A}$ to its maximum value of $425 \mathrm{~A}$ during one control period at motor standstill. This yields a current which is $5.6 \mathrm{~A}$ higher $(1.3 \%$ of the current change) than the precise calculation considering the ohmic resistance. As the effective time constant is much larger in real operation the prediction error there will be even smaller.

The second statement is valid as the flux linkages change linearly in good approximation in real world operation of the machine under test during one control period. As can be seen (a)

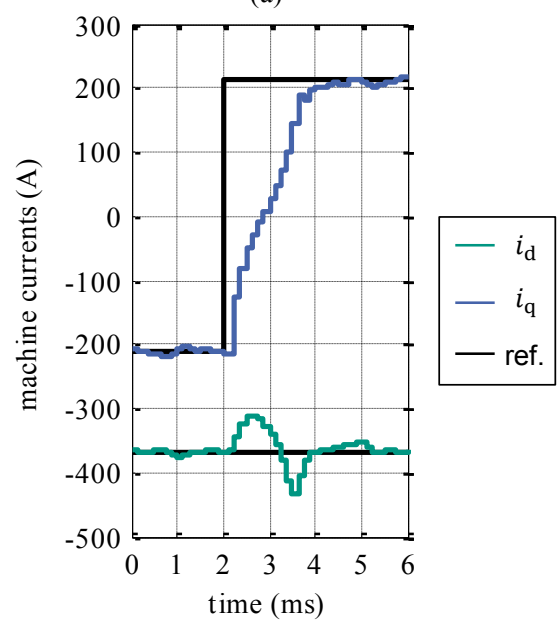

(b)

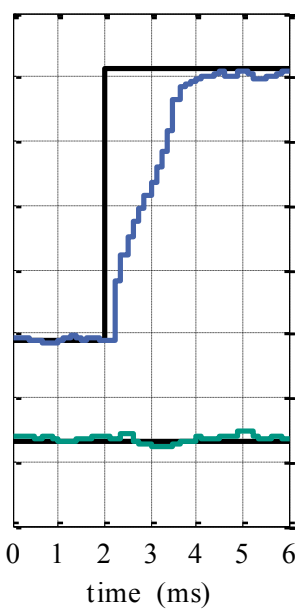

Fig. 6 Torque step measurement from the minimum value of $-220 \mathrm{Nm}$ to the maximum value of $220 \mathrm{Nm}$ at a speed of $2000 \mathrm{~min}^{-1}$ for the PI-type (a) and the proposed controller (b). Nonlinear machine characteristics are clearly visible in the developing of the quadrature current. The proposed controller in contrast to the PI-type controller is able to dynamically decouple the currents of both machine axes as can be seen by the constant direct current in (b).

from the term $-\omega\left(\Psi_{\mathrm{d}}(t)-\Psi_{\mathrm{d}, t_{0}}\right)$ in Fig. 2 the flux linkages develop the more nonlinear the higher the speed is and the more the flux linkages change. A time-step simulation is carried out as a worst-case scenario: The machine is operated at the maximum speed of $11000 \mathrm{~min}^{-1}$ and a torque step from $0 \mathrm{Nm}$ to the maximum value of $220 \mathrm{Nm}$ is performed within one control period of $125 \mu \mathrm{s}$. It should be kept in mind that this torque change cannot be executed with a real machine because a maximal inverter output phase voltage $v_{\max }$ nine times higher than the nominal value of $173.2 \mathrm{~V}$ would be needed. Nevertheless, the currents would change from $0 \mathrm{~A}$ to a direct current $i_{\mathrm{d}, \mathrm{r}}$ of $-368 \mathrm{~A}$ and a quadrature current $i_{\mathrm{q}, \mathrm{r}}$ of $212 \mathrm{~A}$. The precise simulation yields a direct current $4.7 \mathrm{~A}$ lower and a quadrature current $10.8 \mathrm{~A}$ higher than the ones calculated by the current prediction algorithm described in Section II $B$. In total this yields a prediction error of the current amplitude of $11.8 \mathrm{~A}$ or $2.8 \%$ of the amplitude change. During real operation the prediction error will be smaller, typical values are in the order of several amperes and about $0.25 \%$ of the current change.

\section{B. Controller Performance}

The proposed controller is experimentally compared with an advanced PI-type controller that considers the nonlinear machine behavior and the voltage limitation of the inverter. A detailed description of the PI-type controller can be found in [8]. Both controllers are exemplarily analyzed in Fig. 6 on the basis of a torque step from the minimum to the maximum value at a DC link voltage of $300 \mathrm{~V}$ and a speed of $2000 \mathrm{~min}^{-1}$. As can be seen there, the reference value of the direct current does not change while the reference value of the quadrature current inverts. Since both controllers operate at the inverter voltage limit the reference values are reached in the same amount of time. However, the PI-type controller (a) exhibits control deviations of the direct current of about \pm 60 A due to dynamic cross-coupling effects which are not corrected until the quadrature current becomes stationary. As depicted in Fig. 6 (b) these deviations are avoided by the dynamic decoupling 
(a)

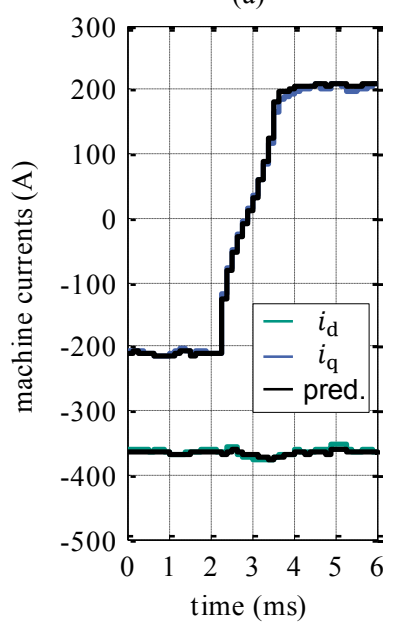

(b)

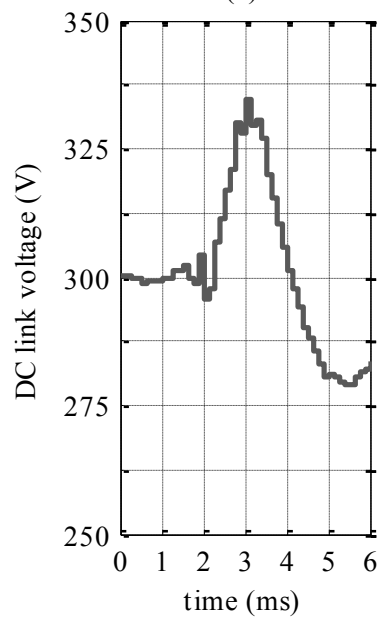

Fig. 7 The predicted currents well suit the measured currents (a). Deviations can be explained by dynamic changes in the DC link voltage (b) and the rotor speed that cannot be accurately measured sufficiently fast.

feature of the proposed control algorithm (see Section III $C$ case 2.1). Hence both machine currents are completely decoupled by the proposed controller even in the presence of transients, saturation and dynamic cross-coupling. The torque inversion is realized as fast as possible in less than $2 \mathrm{~ms}$ omitting unwanted current control deviations.

The predicted and measured direct and quadrature currents for the torque inversion of Fig. 6 are shown in Fig. 7 (a). Current prediction works with good accuracy. Small deviations between the measured and predicted currents are caused by dynamic changes of the machine speed and of the DC link voltage as depicted in Fig. 7 (b). These changes cannot be measured sufficiently fast and therefore yield small model errors.

These errors also prevent the dead-beat step of the proposed controller at the end of the torque inversion as can be seen in Fig. 6 (b): The measured quadrature current slowly approaches the reference value. That is why the dead-beat behavior is analyzed in another test in the nonlinear regime of the machine as given in Fig. 8. There the quadrature current jumps to a lower reference value in generator mode at low speed so that the inverter output voltage is sufficient to perform the current step within one control period. The PI-type controller (a) shows its typical dynamics reaching the reference value within six control periods. The proposed controller however correctly calculates the inverter output voltage using the underlying model and executes the quadrature current change of $75 \mathrm{~A}$ with a dead-beat step within two control periods as depicted in Fig. 8 (b).

\section{SUMMARY}

A control strategy is developed for permanent magnet reluctance torque synchronous machines, taking into account saturation, cross-coupling and the inverter voltage limitation. It is based on current prediction and real-time solving of the machine's nonlinear differential equation system. Thereby complete dynamic decoupling and dead-beat behavior of the currents of both axes is achieved under all operational

(a)

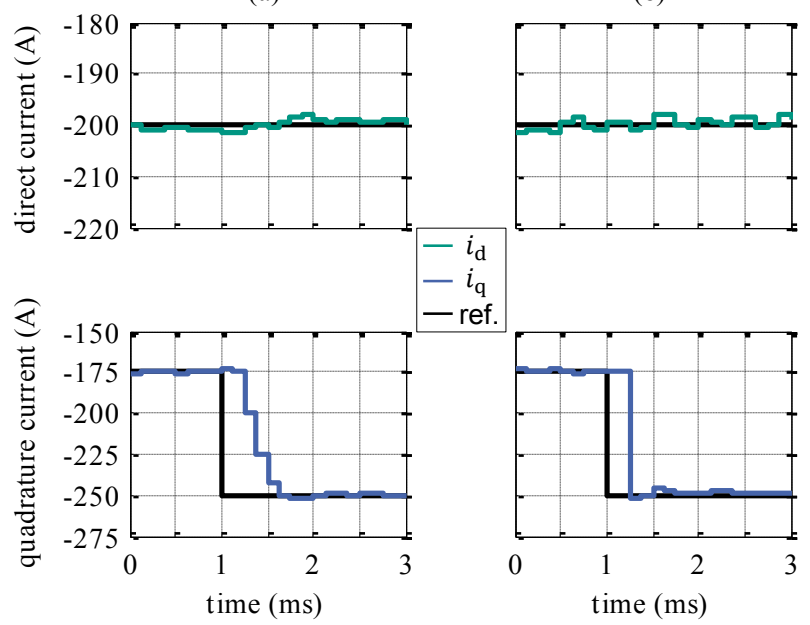

Fig. 8 The quadrature current changes from $-175 \mathrm{~A}$ to $-250 \mathrm{~A}$ at constant direct current and $500 \mathrm{~min}^{-1}$ for the PI-type (a) and the proposed controller (b). Although the machine is strongly nonlinear in the respective current regime the proposed controller shows dead-beat behavior and good decoupling.

conditions although the machine behavior is strongly nonlinear. The controller's performance is experimentally demonstrated by inversion of the maximum torque and a dead-beat current step. The presented methods for real-time solving of the nonlinear machine's equations, current prediction and dynamic decoupling are suitable for application in various control strategies such as trajectory based or model predictive controllers.

\section{REFERENCES}

[1] B. Stumberger, G. Stumberger, D. Dolinar, A. Hamler, and M. Trlep, "Evaluation of saturation and cross-magnetization effects in interior permanent magnet synchronous motor," IEEE Trans. Industry Applications, vol. 39, no. 5, pp. 1264-1271, Sept.-Oct. 2003.

[2] J. Richter, P. Winzer, and M. Doppelbauer, "Application of virtual prototypes of permanent magnet synchronous machines by acausal modeling and simulation," ETG Fachbericht, vol. 139, VDE Verlag GmbH Berlin Offenbach, Nov. 2013.

[3] P. Cortes, M.P. Kazmierkowski, R.M. Kennel, D.E. Quevedo, and J. Rodríguez, "Predictive control in power electronics and drives," IEEE Trans. Industrial Electronics, vol. 55, no. 12, pp. 4312-4324, Dec. 2008.

[4] M.P. Kazmierkowski and L. Malesani, "Current control techiques for three-phase voltage-source PWM converters: a survey," IEEE Trans. Industrial Electronics, vol. 45, no. 5, pp. 691-703, Oct. 1998

[5] F. Morel, X. Lin-Shi, J.M. Rétif, B. Allard, and C. Buttay, "A comparative study of predictive current control schemes for a permanent-magnet synchronous machine drive," IEEE Trans. Industrial Electronics, vol. 56, no. 7, pp. 2715-2728, July 2009

[6] R. Morales-Caporal and M. Pacas, "A predictive torque control for the synchronous reluctance machine taking into account the magnetic cross saturation," IEEE Trans. Industrial Electronics, vol. 54, no. 2, pp. 11611167, April 2007.

[7] J. Weigel and P. Mutschler, "Modelling and control of a permanent magnet linear synchronous motor featuring unbalance and saturation including cross-saturation," in 2004 Power Electronics Specialists Conference, vol. 3, pp. 2204-2210, June 2004.

[8] T. Gemaßmer, J. Richter, and M. Braun, "High dynamic rotor oriented current control for permanent magnet synchronous machines with saturation characteristics," in 2014 PCIM Europe.

[9] J. Seok, J. Kim, and S. Sul, "Overmodulation strategy for highperformance torque control," IEEE Trans. Power Electronics, vol. 13, no. 4, pp. 786-792, July 1998. 